\title{
A Compressible Hamiltonian Electromagnetic Gyrofluid Model
}

\author{
F. L. Waelbroeck \\ Institute for Fusion Studies, University of Texas, Austin, Texas 78712 \\ E. Tassi \\ Centre de Physique Théorique, CNRS Aix-Marseille Universités, \\ Campus de Luminy, case 907, F-13288 Marseille cedex 09, France
}

(Dated: March 15, 2011)

\begin{abstract}
A Lie- Poisson bracket is presented for a four-field gyrofluid model with compressible ions and magnetic field curvature, thereby showing the model to be Hamiltonian. In particular, in addition to commonly adopted magnetic curvature terms present in the continuity equations, analogous terms must be retained also in the momentum equations, in order to have a Lie-Poisson structure. The corresponding Casimir invariants are presented, and shown to be associated to four Lagrangian invariants, that get advected by appropriate "velocity" fields during the dynamics. This differs from a cold ion limit, in which the Lie-Poisson bracket transforms into the sum of direct and semidirect products, leading to only three Lagrangian invariants.

PACS numbers: 52.30.Ex, 52.35.Bj, 94.30.Cq
\end{abstract}




\section{INTRODUCTION}

Fluid models of plasma dynamics play an important role in the investigation of nonlinear phenomena such as turbulence, [1, 2] magnetic reconnection, [3-6] and the propagation of coherent structures. [7-9] A highly desirable property for such models is that they not only have a conserved energy, [10] but also that they be Hamiltonian in the ideal limit. [11] By ideal limit, we mean the limit obtained by neglecting dissipative terms such as those related to collisions and anomalous transport terms.

The Hamiltonian property confers several advantages upon the models that possess it. [12] Krommes and Kolesnikov have shown that the description of the generation of zonal flow and convection cells by short wavelength turbulence depends on the existence of a Poisson bracket. [13] Another important property of noncanonical Hamiltonian systems is the existence of families of invariants, called Casimir invariants, that characterize the dynamics. [12] Adding these Casimirs to the Hamiltonian of the system results in a functional whose stationary points describe equilibrium states and coherent structures. [14] Note, however, that there may exist additional equilibria that do not correspond to stationary points of the Hamiltonian-Casimir functional. This occurs, in particular, at points where the Poisson bracket changes rank. [12]

The existence of non-trivial (e.g. 2D) equilibrium states is essential for physical applications. In the absence of knowledge of the Hamiltonian structure, however, the existence of such equilibrium states can be difficult to ascertain for physically sophisticated models, such as many of the fluid models that are used to describe the dynamics in fusion plasmas (see, for example, [15-21]). Even simple models that otherwise appear physically compelling can fail to be Hamiltonian. For example, Ref. [8] shows that a simple modification of the Hasegawa-Mima model [22], aimed at improving the description of zonal flow dynamics, lacks any equilibrium solution with a non-monotonic electrostatic potential. When a Hamiltonian structure can be identified, by contrast, equilibrium states are readily found.

The stability analysis depends on whether the equilibrium of interest is a stationary point of the Hamiltonian-Casimir functional. In the affirmative, convexity arguments may serve to demonstrate nonlinear stability. When convexity cannot be established, the analysis of the second variation of the Hamiltonian-Casimir functional may still provide information about the "energy" stability (which implies linear stability) [23]. For more general equilib- 
ria, stability can be investigated for perturbations that conserve all the Casimir invariants, sometimes referred to as "dynamically accessible" perturbations. [23] The "Energy principle" of ideal magnetohydrodynamics is a well-known example of this.

Irrespective of the utility of Hamiltonian formulations, there is a fundamental argument for their necessity. All plasma models aim to describe charged particles interacting with electromagnetic fields, a system that obeys Hamiltonian dynamics. It is thus desirable that any reduced description of this system inherit a Hamiltonian substructure.

An important problem in plasma physics is the description of phenomena such that the effects of finite ion temperature plays a central role. This includes, for example, magnetohydrodynamic instabilities such that the growth rate is comparable to the ion diamagnetic drift frequency and localized modes with wavelengths across the field that are comparable to the ion Larmor radius. The construction of models that account for the effects of ion temperature, have a form compatible with the gyroviscous cancellations and satisfy the Hamiltonian property is difficult. For instance, the four-field model of Ref. [15] conserves energy, but efforts to identify its Hamiltonian structure have been unsuccessful. Hazeltine et al. [24] have constructed an alternative Hamiltonian model by using a mapping technique to introduce finite ion temperature into the cold ion limit of the model of Ref. [15], for which a Poisson bracket is known. The resulting system, known as the Hamiltonian four-field finite Larmor radius (FLR) model, describes the evolution of the density, the parallel ion velocity, and the magnetic and electrostatic potentials. Unfortunately, their model involves third-order derivatives of the current and the vorticity, raising questions as to the nature of suitable boundary conditions. Perhaps because of this, it has not been applied or studied further since its inception.

A few years after the formulation of the four-field model, research on turbulent ion thermal transport in fusion experiments led to the discovery of a new class of fluid models obtained by truncating the moment hierarchy of the gyro-averaged kinetic equation, instead of the original kinetic equation. [16-18, 21] These models, known as gyro-fluid models, rely on nonlocal operators to describe the effect of Larmor gyration on the response of the ions. As a result, they provide far superior approximations to the kinetic dispersion relation for linear waves. In particular, they describe accurately the propagation of disturbances with wavelengths smaller than the Larmor radius, in contrast with so-called FLR (Finite Larmor Radius) models, such as the four-field model of Ref. [24], that are based on the assumption 
that the wavelengths are much longer than the Larmor radius.

In the present paper, we construct a noncanonical Hamiltonian formulation for a simple electromagnetic gyrofluid model. Like the model studied in our previous work, [25] the model that we investigate in the present paper is a truncation of the much more complete model proposed by Snyder and Hammett that advances 6 moments for the ions and two moments for the electron dynamics. [21] Two new features in the present work are that (1) we retain the effect of ion compressibility, thereby enabling the description of sound waves and drift Kelvin-Helmholtz instabilities [26] and (2) we account for the role of magnetic curvature, thereby enabling our model to describe geodesic acoustic modes (GAM).[27, 28]

The present paper is organized as follows. Sec. II introduces the dynamical model that we wish to find a Poisson bracket for, and presents the energy. In Sec. III we construct the Poisson bracket for this model. In Sec. IV we calculate the Casimir invariants, and in Sec. 25 we introduce the normal variables suggested by the Casimirs, and use these normal variables to show that the Poisson bracket satisfies the Jacobi identity. Lastly, we summarize our findings in Sec. VI. Appendix A contains an explicit proof of the Jacobi identity for our Poisson bracket.

\section{FORMULATION}

We are interested in a model that describes the interaction of kinetic Alfvén waves with drift-acoustic waves. In order to describe both the "inertial" $\left(\beta_{e} \ll m_{e} / m_{i}\right)$ and the "kinetic" $\left(\beta_{e} \gg m_{e} / m_{i}\right)$ regimes of the Alfvén wave, we leave $\beta_{e}$ unrestricted. [29] $\left(\beta_{e}\right.$ is the ratio of the electron kinetic pressure to the magnetic pressures). For $\beta_{e} \sim m_{e} / m_{i}\left(v_{t e} \sim v_{A}\right)$, however, our equations must be modified to account for electron Landau damping. We consider the evolution of the density $n_{i}$ and the parallel velocity of the ion guiding centers $u_{i}=\hat{\mathbf{z}} \cdot \mathbf{v}_{i}$, the electron density $n_{e}$ and parallel velocity $u_{e}=\hat{\mathbf{z}} \cdot \mathbf{v}_{e}$, and the magnetic flux $A=\hat{\mathbf{z}} \cdot \mathbf{A}$ where $\mathbf{A}$ is the vector potential. The quasi-neutrality condition determines the electrostatic potential $\phi$. We normalize these quantities according to

$$
\left(n_{i}, n_{e}, A, \phi, u_{i}, u_{e}\right)=\frac{L_{n}}{\rho_{s}}\left(\frac{\hat{n}_{i}}{n_{0}}, \frac{\hat{n}_{e}}{n_{0}}, \frac{\hat{A}_{z}}{\rho_{s} B_{0}}, \frac{e \hat{\phi}}{T_{e 0}}, \frac{\hat{v}_{i}}{c_{s}}, \frac{\hat{v}_{e}}{c_{s}}\right),
$$

where the carets denote the dimensional variables. Here $n_{0}$ is the background density, $\rho_{s}=c_{s} / \omega_{c i}$ where $c_{s}=\left(T_{e} / m_{i}\right)^{1 / 2}$ is the sound speed, $\omega_{c i}=e B_{0} / m_{i}$ is the ion cyclotron 
frequency, and $L_{n}=n_{0} /|\nabla n|$ is the density scale-length. We also normalize the independent variables according to

$$
\left(t, k_{\|}, k_{\perp}\right)=\left(\hat{t} c_{s} / L_{n}, \hat{k}_{\|} L_{n}, \hat{k}_{\perp} \rho_{s}\right) .
$$

Normalizing the perpendicular lengths to $\rho_{s}$ has the advantage of making the $\tau=T_{i} / T_{e} \rightarrow 0$ limit transparent.

We obtain our evolution equations from the equations of Ref. [21] by assuming constant temperatures and discarding all but the lowest two parallel moments for the ions and electrons. We also discard all the magnetic pumping terms, proportional to $\nabla_{\|} \log B$, which involve transfers between the parallel and perpendicular thermal energy. The equations that follow from these choices are

$$
\begin{array}{r}
\frac{\partial n_{s}}{\partial t}+\left[\Phi_{s}, n_{s}\right]+2 v_{d}\left[x, \tau_{s} n_{s} \pm \Phi_{s}\right]+\nabla_{\| s} u_{s}=0 \\
\mu_{s}\left(\frac{\partial u_{s}}{\partial t}+\left[\Phi_{s}, u_{s}\right]\right) \mp E_{\| s}+\tau_{s} \nabla_{\| s} n_{s}+2 \lambda \mu_{s} \tau_{s} v_{d}\left[x, u_{s}\right]=0
\end{array}
$$

where the upper (lower) sign should be taken for the ions (electrons), $s=i$, e denotes the species index, $\tau_{s}=T_{s} / T_{e}, \mu_{s}=m_{s} / m_{i}, v_{d}$ is the curvature drift and $\lambda$ is a numerical coefficient that is calculated to be 2 in Ref. [21]. We will see below that in order for the two-moment system to be Hamiltonian, only half of this term should be retained: that is, one must choose $\lambda=1$. The same conclusion was reached for the FLR version of the fourfield model in Ref. [24], as can be seen by comparing the curvature term in the Hamiltonian version of the parallel momentum equation to that for the version obtained through an FLR expansion in Ref. [30].

The remaining symbols in the above equations are the parallel gradient, $\nabla_{\| s} \xi=\partial_{z} \xi+$ $\left[\xi, \mathcal{A}_{s}\right]$ for any field $\xi$, and the electric field,

$$
E_{\| s}=-\frac{\partial \mathcal{A}_{s}}{\partial t}-\nabla_{\| s} \Phi_{s}
$$

The fields $\Phi_{s}$ and $\mathcal{A}_{s}$ are the gyroaverages of $\phi$ and $A$. We will neglect the gyroradius of the electrons, so that $\Phi_{e}=\phi$ and $\mathcal{A}_{e}=A$. For the ions, by contrast, we take

$$
\begin{gathered}
\Phi_{i}=\Gamma_{0}^{1 / 2} \phi \equiv \Phi \\
\mathcal{A}_{i}=\Gamma_{0}^{1 / 2} A \equiv \mathcal{A} .
\end{gathered}
$$

Here $\Gamma_{0}^{1 / 2}$ is an operator introduced by Dorland and Hammett [18] and defined by

$$
\Gamma_{0}^{1 / 2} \xi=\exp \left(\frac{1}{2} \tau \nabla_{\perp}^{2}\right) I_{0}^{1 / 2}\left(-\tau \nabla_{\perp}^{2}\right) \xi
$$


where $I_{0}$ is the modified Bessel function of the first kind and $\tau=\tau_{i}=T_{i} / T_{e}$. The definition in Eq. (5) should be interpreted in terms of its series expansion

$$
\Gamma_{0}^{1 / 2} \xi=1+\sum_{n=1}^{\infty} a_{n}\left(\tau \nabla_{\perp}^{2}\right)^{n}=1+(\tau / 2) \nabla^{2}+\cdots
$$

where the $a_{n}$ are real numbers. In collocation-based numerical solutions, the Bessel functions in Eq. (5) may be evaluated to required accuracy. For finite difference codes, by contrast, it is generally preferable to use a Padé approximant, such as

$$
\Gamma_{0}^{1 / 2}(b)=(1+b / 2)^{-1}
$$

in order to simplify the necessary calculations of $\Gamma_{0}, \Gamma_{0}^{1 / 2}$, and their inverses. Ref. [18] provides a comparison of the accuracy of a selection of approximants of various orders.

The system is completed by the quasi-neutrality equation,

$$
n_{e}=\Gamma_{0}^{1 / 2} n_{i}+\left(\Gamma_{0}-1\right) \phi / \tau,
$$

where $\Gamma_{0}=\left(\Gamma_{0}^{1 / 2}\right)^{2}$, and by Ampére's law,

$$
\nabla^{2} A=\frac{\beta_{e}}{2}\left(u_{e}-\Gamma_{0}^{1 / 2} u_{i}\right)
$$

To facilitate comparison to earlier models including the cold-ion version of the four-field model [31] and the incompressible (3-field) gyrofluid model, [25] we rewrite the dynamical equations as

$$
\begin{aligned}
\frac{\partial n_{i}}{\partial t}+\left[\Phi, n_{i}\right]+2 v_{d}\left[x, \tau n_{i}+\Phi\right]+\partial_{z} u_{i}+\left[u_{i}, \mathcal{A}\right] & =0 ; \\
\frac{\partial \mathcal{M}_{i}}{\partial t}+\left[\Phi, \mathcal{M}_{i}\right]+\partial_{z}\left(\tau n_{i}+\Phi\right)+\tau\left[n_{i}, \mathcal{A}\right]+2 \lambda \tau v_{d}\left[x, u_{i}\right] & =0 ; \\
\frac{\partial n_{e}}{\partial t}+\left[\phi, n_{e}\right]-2 v_{d}\left[x, n_{e}-\phi\right]+\partial_{z} u_{e}+\left[u_{e}, A\right] & =0 ; \\
\frac{\partial \mathcal{M}_{e}}{\partial t}+\left[\phi, \mathcal{M}_{e}\right]+\partial_{z}\left(n_{e}-\phi\right)+\left[n_{e}, A\right]-2 \lambda \mu v_{d}\left[x, u_{e}\right] & =0 ;
\end{aligned}
$$

where $\mathcal{M}_{i}=u_{i}+\mathcal{A}$ and $\mathcal{M}_{e}=\mu u_{e}-A$, with $\mu=\mu_{e}=m_{e} / m_{i}$, are respectively the ion and electron momenta.

The above equations conserve the following energy:

$$
H=\frac{1}{2}\left\langle\tau n_{i}^{2}+u_{i}^{2}+n_{e}^{2}+\mu u_{e}^{2}+\frac{2}{\beta_{e}}|\nabla A|^{2}+\Phi n_{i}-\phi n_{e}\right\rangle .
$$


where we have used the quasi-neutrality equation to simplify the result. Here, the angular brackets denote the integral over the volume of interest, $\langle g(x, y, z, t)\rangle=$ $\iiint g(x, y, z, t) d x d y d z$, and the boundary conditions have been assumed to be such that the surface integrals vanish. The successive terms represent, respectively, the ion thermal and kinetic energies, the electron thermal and kinetic energies, the magnetic energy, and the electrostatic energy of the ions and electrons. We note that $H$ is conserved regardless of the value of $\lambda$.

\section{POISSON BRACKET}

We adopt $n_{i}, \mathcal{M}_{i}, n_{e}$, and $\mathcal{M}_{e}$ as our dynamical variables. In terms of these variables the flux $A$ is given by Ampére's law and the potential $\phi$ by the quasi-neutrality condition,

$$
\phi=\tau\left(\Gamma_{0}-1\right)^{-1}\left(n_{e}-\Gamma_{0}^{1 / 2} n_{i}\right)
$$

The variations of the Hamiltonian are

$$
\begin{aligned}
H_{n_{i}} & =\tau n_{i}+\Phi ; \\
H_{\mathcal{M}_{i}} & =u_{i} ; \\
H_{n_{e}} & =n_{e}-\phi ; \\
H_{\mathcal{M}_{e}} & =u_{e}
\end{aligned}
$$

where $H_{\xi_{j}}$ is a shorthand notation for the functional derivative of $H\left[\xi_{1}, \xi_{2}, \xi_{3}, \xi_{4}\right]$ with respect to the fields $\xi_{1}=n_{i}, \xi_{2}=\mathcal{M}_{i}, \xi_{3}=n_{e}, \xi_{4}=\mathcal{M}_{e}$ :

$$
H_{\xi_{j}}:=\frac{\delta H}{\delta \xi_{j}}, \quad j=1,2,3,4 .
$$

We may write the equations of motion in terms of the variations of $H$ as follows:

$$
\begin{aligned}
\frac{\partial n_{i}}{\partial t} & =-\partial_{z} H_{\mathcal{M}_{i}}+\left[n_{i}-2 v_{d} x, H_{n_{i}}\right]+\left[\mathcal{M}_{i}, H_{\mathcal{M}_{i}}\right] \\
\frac{\partial \mathcal{M}_{i}}{\partial t} & =-\partial_{z} H_{n_{i}}+\tau\left[n_{i}-2 \lambda v_{d} x, H_{\mathcal{M}_{i}}\right]+\left[\mathcal{M}_{i}, H_{n_{i}}\right] \\
\frac{\partial n_{e}}{\partial t} & =-\partial_{z} H_{\mathcal{M}_{e}}-\left[n_{e}-2 v_{d} x, H_{n_{e}}\right]-\left[\mathcal{M}_{e}, H_{\mathcal{M}_{e}}\right] ; \\
\frac{\partial \mathcal{M}_{e}}{\partial t} & =-\partial_{z} H_{n_{e}}-\mu\left[n_{e}-2 \lambda v_{d} x, H_{\mathcal{M}_{e}}\right]-\left[\mathcal{M}_{e}, H_{n_{e}}\right]
\end{aligned}
$$


The corresponding Poisson bracket is

$$
\{F, G\}=\sum_{s=i, e}\left(\{F, G\}_{\| s}+\{F, G\}_{\perp s}\right)
$$

where

$$
\{F, G\}_{\| s}=-\left\langle F_{n_{s}} \partial_{z} G_{\mathcal{M}_{s}}-G_{n_{s}} \partial_{z} F_{\mathcal{M}_{s}}\right\rangle
$$

and

$$
\begin{gathered}
\{F, G\}_{\perp s}=\mp\left\langle\left(n_{s}-2 v_{d} x\right)\left[F_{n_{s}}, G_{n_{s}}\right]+\mu_{s} \tau_{s}\left(n_{s}-2 \lambda v_{d} x\right)\left[F_{\mathcal{M}_{s}}, G_{\mathcal{M}_{s}}\right]\right\rangle \\
\mp\left\langle\mathcal{M}_{s}\left(\left[F_{\mathcal{M}_{s}}, G_{n_{s}}\right]+\left[F_{n_{s}}, G_{\mathcal{M}_{s}}\right]\right)\right\rangle,
\end{gathered}
$$

where the $\mp$ has the same meaning as in Eq. 2

In Sec. V, we will show that for $\lambda=1$, this Poisson bracket satisfies the Jacobi identity,

$$
\mathcal{J}:=\{F,\{G, H\}\}+\{G,\{H, F\}\}+\{H,\{F, G\}\}=0
$$

for all functionals $F, G, H$.

\section{INVARIANTS}

The Poisson brackets characterize the geometrical properties of the dynamics that are independent from the Hamiltonian. Perhaps the most important of these properties is the existence of Casimir functionals, which are invariants of the motion that specify a foliation of phase space. [23, 32] In the presence of an ignorable coordinate, these invariants usually appear in infinite families and become useful to construct variational principles for studying the equilibrium and stability of a system. In this section we assume $\partial_{z}=0$ : the generalization to the case of helical symmetry is straightforward and is described in Ref. [32].

We obtain the Casimir functional $C\left[n_{i}, \mathcal{M}_{i}, n_{e}, \mathcal{M}_{e}\right]$ by expressing the condition that $\left\{\xi_{j}, C\right\}=0$ for $\xi_{1 s}=n_{s}, \xi_{2 s}=\mathcal{M}_{s}$. This results in the following system of equations:

$$
\begin{array}{r}
{\left[n_{s}-2 x v_{d}, C_{n_{s}}\right]+\left[\mathcal{M}_{s}, C_{\mathcal{M}_{s}}\right]=0 ;} \\
\mu_{s} \tau_{s}\left[n_{s}-2 \lambda x v_{d}, C_{\mathcal{M}_{s}}\right]+\left[\mathcal{M}_{s}, C_{n_{s}}\right]=0 ;
\end{array}
$$

Solving the first equation, Eq. (26), yields $C=\sum_{s} G_{s}\left[n_{s}, \mathcal{M}_{s}\right]=\sum_{s}\left\langle g_{s}\left(n_{s}-2 x v_{d}, \mathcal{M}_{s}\right)\right\rangle$ where the $g_{s}$ are arbitrary functions. Substituting this result in the second equation, Eq. (27), 
we find that there is no well-behaved solution except if $\lambda=1$. In the Appendix we show that indeed, for $\lambda \neq 1$, the bracket (24) does not satisfy the Jacobi identity and thus is not a Poisson bracket. Considering then the case $\lambda=1$, we obtain

$$
\mu_{s} \tau_{s} \partial_{\mathcal{M}_{s}}^{2} g_{s}\left(n_{s}-2 x v_{d}, \mathcal{M}_{s}\right)-\partial_{n_{s}}^{2} g_{s}\left(n_{s}-2 x v_{d}, \mathcal{M}_{s}\right)=0
$$

Integrating this using the method of characteristics, we find two pairs of independent solutions $G_{s}=G_{s \pm}\left[n_{s}, \mathcal{M}_{s}\right]$ where

$$
G_{s \pm}\left[n_{s}, \mathcal{M}_{s}\right]=\left\langle g_{s \pm}\left(\mathcal{M}_{s} \pm \sqrt{\mu_{s} \tau_{s}}\left(n_{s}-2 x v_{d}\right)\right)\right\rangle
$$

and the $g_{s \pm}$ are two new arbitrary functions. The general form of the Casimir functional for $\lambda=1$ is thus

$$
C\left[n_{i}, \mathcal{M}_{i}, n_{e}, \mathcal{M}_{e}\right]=\sum_{s, \pm}\left\langle g_{s+}\left(\mathcal{M}_{s} \pm \sqrt{\mu_{s} \tau_{s}}\left(n_{s}-2 x v_{d}\right)\right)\right\rangle .
$$

We will henceforth restrict consideration to the case $\lambda=1$.

\section{NORMAL FIELDS AND JACOBI}

The form of the Casimirs suggests the introduction of a new set of variables, which we call "normal fields":

$$
\mathcal{V}_{s \pm}=\mathcal{M}_{s} \pm \sqrt{\tau_{s} \mu_{s}}\left(n_{s}-2 x v_{d}\right)
$$

In terms of these normal fields, the equations of motion take the form

$$
\frac{\partial \mathcal{V}_{s \pm}}{\partial t}+\left[\Phi_{s \pm}, \mathcal{V}_{s \pm}\right]=0
$$

where

$$
\Phi_{s \pm}=\Phi_{s} \mp \mathcal{A}_{s} \sqrt{\tau_{s} / \mu_{s}}
$$

are stream-functions describing the flows that convect the fields $\mathcal{V}_{s \pm}$. The latter are thus Lagrangian conserved quantities. Grasso et al. [33] have shown that the mixing of such Lagrangian quantities by the convecting flows is an important ingredient in rapid collisionless reconnection. In this respect, it is worth noticing that a limit of this model [31], obtained by neglecting the ion gyroradius, possesses a Poisson bracket of a different type (the sum of direct and semidirect products), leading to only three Lagrangian invariants, two of which undergoing phase mixing. 
The Poisson bracket of (22) can be written in terms of the normal fields. Dropping the species subscripts for clarity, the functional derivatives take the form

$$
\begin{aligned}
F_{n} & =\left(F_{\mathcal{V}_{+}}-F_{\mathcal{V}_{-}}\right) \sqrt{\mu \tau} \\
F_{\mathcal{M}} & =F_{\mathcal{V}_{+}}+F_{\mathcal{V}_{-}}
\end{aligned}
$$

Inserting these functional derivatives into the brackets of (23) and (24) gives

$$
\{F, G\}_{\|}=2 \sqrt{\mu \tau} \sum_{ \pm} \pm\left\langle F_{\mathcal{V}_{+}} \partial_{z} G_{\mathcal{V}_{+}}\right\rangle
$$

and

$$
\{F, G\}_{\perp}=\frac{2}{\sqrt{\mu \tau}} \sum_{ \pm}\left\langle\mathcal{V}_{ \pm}\left[F_{\mathcal{V}_{ \pm}}, G_{\mathcal{V}_{ \pm}}\right]\right\rangle
$$

The above sums of independent brackets of the four variables are known as a direct product. [34] Since the individual brackets satisfy the Jacobi identity, their sums in Eqs. (34) and (35) also do. By virtue of the coordinate invariance of the Jacobi identity, it follows that the parallel and perpendicular brackets given by Eqs. (23) and (24) also satisfy the Jacobi identity.

As stated in Sec. I, the identification of a Hamiltonian structure in the presence of both magnetic inhomogeneities and finite ion temperature is difficult, even if a conserved quantity for the model is known. [35] For instance, if we had retained the magnetic curvature terms in the continuity equations (1), but neglected them in the momentum equations (2), as done in the partially linearized model of Ref. [19], the functional (12) would still have been a conserved quantity, but the resulting bracket obtained from (22) would have no longer satisfied the Jacobi identity.

\section{SUMMARY}

We have presented a Poisson bracket structure for the four-field version of the gyrofluid equations of Snyder and Hammett, [21] Eqs. (1)-(2), that we obtained by keeping only the first two moments of the gyrokinetic equations for both the electrons and the ions. We find that the system is Hamiltonian provided that the coefficient of the curvature term in the parallel momentum equation is halved from the value obtained in the six-moment expansion of Ref. [21]. We note that Hazeltine et al. [24] reached a similar conclusion in the long 
wavelength (FLR) limit. One may speculate that the omitted part of the curvature term contributes to the Poisson bracket for a more complete system that includes the contributions from the action of the curvature on the heat fluxes.

With the modification noted above, the bracket admits a Casimir functional, given in Eq. (29), that depends on four so-called normal fields, which are linear combinations of the canonical momentum densities and the guiding center densities. The dynamical equations for the normal fields take the form of simple Lagrangian convection equations, with convecting flows that derive from four different stream functions given by Eq. (31). The existence of a proper Poisson bracket describing the dynamics guarantees that the equilibrium equations obtained by neglecting the time derivatives (possibly in a frame of reference moving at constant velocity) may be integrated once to obtain Grad-Shafranov-like equations that determine the magnetic flux (i.e. the component of the vector-potential along the background field) and the electrostatic potential.

Possible applications of the electromagnetic gyrofluid model presented here include any electromagnetic problems where sound waves, diamagnetic drifts and nonlinear effects are important. This includes, in particular, the problem of the mutual interaction of magnetic islands and resonant magnetic perturbations with plasma turbulence. [36, 37]

\section{APPENDIX A: VIOLATION OF THE JACOBI IDENTITY FOR $\lambda \neq 1$}

In Secs. IV and 25 we showed that, for $\lambda=1$, the bracket (24) is a Poisson bracket, because it can be mapped to the direct product form, which is known to satisfy all the properties of a Poisson bracket, and in in particular the Jacobi identity. In the following we show that, if $\lambda \neq 1$, the Jacobi identity is violated, thus leaving $\lambda=1$ as the only admissible choice for obtaining a Hamiltonian structure.

Given that the bracket (24) is a linear combination of two independent bilinear forms, one for ions and one for electrons, we consider, only the electron part

$$
\{F, G\}_{\perp e}=\left\langle\left(n_{e}-2 v_{d} x\right)\left[F_{n_{e}}, G_{n_{e}}\right]+\mu\left(n_{e}-2 \lambda v_{d} x\right)\left[F_{\mathcal{M}_{e}}, G_{\mathcal{M}_{e}}\right]\right\rangle+\left\langle\mathcal{M}_{e}\left(\left[F_{\mathcal{M}_{e}}, G_{n_{e}}\right]+\left[F_{n_{e}}, G_{\mathcal{M}_{e}}\right]\right)\right\rangle
$$

although the same arguments can of course be applied to the ion part. 
The goal is to show that

$$
\left\{\{F, G\}_{\perp e}, H\right\}_{\perp e}+\left\{\{G, H\}_{\perp e}, F\right\}_{\perp e}+\left\{\{H, F\}_{\perp e}, G\right\}_{\perp e}=0
$$

for all functionals $F, G, H$, is not satisfied if $\lambda \neq 1$. We first remark that

$$
\begin{gathered}
\frac{\delta\{F, G\}_{\perp e}}{\delta n_{e}}=\left[F_{n_{e}}, G_{n_{e}}\right]+\mu\left[F_{\mathcal{M}_{e}}, G_{\mathcal{M}_{e}}\right]+t . i . s . d . \\
\frac{\delta\{F, G\}_{\perp e}}{\delta \mathcal{M}_{e}}=\left[F_{M_{e}}, G_{n_{e}}\right]+\left[F_{n_{e}}, G_{M_{e}}\right]+t . i . s . d .
\end{gathered}
$$

where t.i.s.d. means "terms involving second derivatives". Using these expressions, we can rewrite the first term of (A2) as

$$
\begin{aligned}
& \left\{\{F, G\}_{\perp e}, H\right\}_{\perp e}=\left\langle\left(n_{e}-2 v_{d} x\right)\left[\left[F_{n_{e}}, G_{n_{e}}\right]+\mu\left[F_{\mathcal{M}_{e}}, G_{\mathcal{M}_{e}}\right], H_{n_{e}}\right]+\right. \\
& \mu\left(n_{e}-2 \lambda v_{d} x\right)\left[\left[F_{\mathcal{M}_{e}}, G_{n_{e}}\right]+\left[F_{n_{e}}, G_{\mathcal{M}_{e}}\right], H_{\mathcal{M}_{e}}\right]+ \\
& \mathcal{M}_{e}\left(\left[\left[F_{\mathcal{M}_{e}}, G_{n_{e}}\right]+\left[F_{n_{e}}, G_{\mathcal{M}_{e}}\right], H_{n_{e}}\right]+\left[\left[F_{n_{e}}, G_{n_{e}}\right]+\mu\left[\left[F_{\mathcal{M}_{e}}, G_{\mathcal{M}_{e}}\right], H_{\mathcal{M}_{e}}\right]\right)+\text { t.i.s.d. }\right\rangle .
\end{aligned}
$$

Note now that, in order for (A2) to hold, all the terms containing explicitly, $n_{e}, x, \mathcal{M}_{e}$ and second functional derivatives, respectively, have to group together and vanish separately. Let us focus on the terms depending explicitly on $x$. We have

$$
\begin{aligned}
& \left\{\{F, G\}_{\perp e}, H\right\}_{\perp e}+\left\{\{G, H\}_{\perp e}, F\right\}_{\perp e}+\left\{\{H, F\}_{\perp e}, G\right\}_{\perp e}= \\
& \left\langle-2 v_{d} x\left(\left[\left[F_{n_{e}}, G_{n_{e}}\right]+\mu\left[F_{\mathcal{M}_{e}}, G_{\mathcal{M}_{e}}\right], H_{n_{e}}\right]+\left[\left[G_{n_{e}}, H_{n_{e}}\right]+\mu\left[G_{\mathcal{M}_{e}}, H_{\mathcal{M}_{e}}\right], F_{n_{e}}\right]+\right.\right. \\
& \left.\left[\left[H_{n_{e}}, F_{n_{e}}\right]+\mu\left[H_{\mathcal{M}_{e}}, F_{\mathcal{M}_{e}}\right], G_{n_{e}}\right]\right)+ \\
& \mu \lambda\left(\left[\left[F_{\mathcal{M}_{e}}, G_{n_{e}}\right]+\left[F_{n_{e}}, G_{\mathcal{M}_{e}}\right], H_{\mathcal{M}_{e}}\right]+\left[\left[G_{\mathcal{M}_{e}}, H_{n_{e}}\right]+\left[G_{n_{e}}, H_{\mathcal{M}_{e}}\right], F_{\mathcal{M}_{e}}\right]+\right. \\
& \left.\left.\left[\left[H_{\mathcal{M}_{e}}, F_{n_{e}}\right]+\left[H_{n_{e}}, F_{\mathcal{M}_{e}}\right], G_{\mathcal{M}_{e}}\right]\right)+\cdots\right\rangle= \\
& \left\langle-2 v_{d} x\left(\left[\left[F_{n_{e}}, G_{n_{e}}\right], H_{n_{e}}\right]+\left[\left[G_{n_{e}}, H_{n_{e}}\right], F_{n_{e}}\right]+\left[\left[H_{n_{e}}, F_{n_{e}}\right], G_{n_{e}}\right]+\right.\right. \\
& \mu\left(\left[\left[F_{\mathcal{M}_{e}}, G_{\mathcal{M}_{e}}\right], H_{n_{e}}\right]+\lambda\left[\left[G_{\mathcal{M}_{e}}, H_{n_{e}}\right], F_{\mathcal{M}_{e}}\right]+\lambda\left[\left[H_{n_{e}}, F_{\mathcal{M}_{e}}\right], G_{\mathcal{M}_{e}}\right]\right)+ \\
& \left.\mu\left(\left[\left[G_{\mathcal{M}_{e}}, H_{\mathcal{M}_{e}}\right], F_{n_{e}}\right]+\lambda\left[\left[F_{n_{e}}, G_{\mathcal{M}_{e}}\right], H_{\mathcal{M}_{e}}\right]+\lambda\left[\left[H_{\mathcal{M}_{e}}, F_{n_{e}}\right], G_{\mathcal{M}_{e}}\right]\right)\right)+ \\
& \left.\mu\left(\left[\left[H_{\mathcal{M}_{e},}, F_{\mathcal{M}_{e}}\right], G_{n_{e}}\right]+\lambda\left[\left[G_{n_{e}}, H_{\mathcal{M}_{e}}\right], F_{\mathcal{M}_{e}}\right]+\lambda\left[\left[F_{\mathcal{M}_{e}}, G_{n_{e}}\right], H_{\mathcal{M}_{e}}\right]\right)+\cdots\right\rangle,
\end{aligned}
$$

where the dots indicate all the terms depending explicitly on $n_{e}, \mathcal{M}_{e}$ and on the second functional derivatives. From the last expression one can see that the sum of three terms containing $F_{n_{e}}, G_{n_{e}}$ and $H_{n_{e}}$ vanishes by virtue of the Jacobi identity for the inner bracket $[$,$] . Applying the "inner Jacobi" identity to the terms in the last two lines of (A6), however,$ 
we find

$$
\begin{aligned}
& \left\{\{F, G\}_{\perp e}, H\right\}_{\perp e}+\left\{\{G, H\}_{\perp e}, F\right\}_{\perp e}+\left\{\{H, F\}_{\perp e}, G\right\}_{\perp e} \\
& \quad=\left\langle\mu(\lambda-1) 2 v_{d} x\left(\left[\left[F_{\mathcal{M}_{e}}, G_{\mathcal{M}_{e}}\right], H_{n_{e}}\right]+\left[\left[G_{\mathcal{M}_{e}}, H_{\mathcal{M}_{e}}\right], F_{n_{e}}\right]\right)+\left[\left[H_{\mathcal{M}_{e}}, F_{\mathcal{M}_{e}}\right], G_{n_{e}}\right]+\cdots \chi \mathrm{A} 7\right)
\end{aligned}
$$

We see that these terms vanish for all $F, G$ and $H$ only if $\lambda=1$. For $\lambda \neq 1$ then, the form (24) is not a Poisson bracket.

\section{ACKNOWLEDGEMENTS}

E.T. acknowledges useful discussions with Olivier Izacard. This work was supported by the US DOE under contracts DE-FG03-96ER-54346, and by the European Community under the contracts of Association between EURATOM, CEA, and the French Research Federation for fusion studies. The views and opinions expressed herein do not necessarily reflect those of the European Commission. E.T. acknowledges also the financial support from the Agence Nationale de la Recherche (ANR GYPSI).

[1] T. Passot and P. L. Sulem, Physics of Plasmas 14, 082502 (2007).

[2] B. D. Scott, Plasma Phys. Control. Fusion 49, S25 (2007).

[3] D. Grasso, F. Califano, F. Pegoraro, and F. Porcelli, Plasma Physics Reports 26, 512 (2000).

[4] B. N. Rogers, R. E. Denton, J. F. Drake, and M. A. Shay, Phys. Rev. Lett. 87, 195004 (2001).

[5] E. Tassi, F. L. Waelbroeck, and D. Grasso, Journal of Physics: Conference Series 260, 012020 (2010).

[6] D. Grasso, E. Tassi, and F. L. Waelbroeck, Physics of Plasmas 17, 082312 (2010).

[7] S. I. Krasheninnikov, Phys. Letters A 283, 368 (2001).

[8] F. L. Waelbroeck, P. J. Morrison, and W. Horton, Plasma Physics and Controlled Fusion 46, 1331 (2004).

[9] O. Alexandrova, A. Mangeney, M. Maksimovic, N. Cornilleau-Wehrlin, J. M. Bosqued, and M. Andre, J. Geophys. Res. - Space Phys. 111 (2006).

[10] B. D. Scott, Phys. Plasmas 12, 102307 (2005).

[11] P. J. Morrison, Phys. Plasmas 12, 058102 (2005). 
[12] P. J. Morrison, Poisson brackets for fluids and plasmas, in Mathematical Methods in Hydrodynamics and Integrability in Dynamical Systems, edited by M. Tabor and Y. Treve, volume 88 of American Institute of Physics Conference Proceedings, pp. 13-45, American Institute of Physics, 1982.

[13] J. A. Krommes and R. A. Kolesnikov, Phys. Plasmas 11, L29 (2004).

[14] P. J. Morrison and M. Kotschenreuther, The free energy principle, negative energy modes, and stability, in Nonlinear World: IV International Workshop on Nonlinear and Turbulent Processes in Physics, edited by V. G. Baryakhtar, V. M. Chernousenko, N. S. Erokhin, A. B. Sitenko, and V. E. Zakharov, World Scientific, 1990.

[15] R. D. Hazeltine, M. Kotschenreuther, and P. J. Morrison, Phys. Fluids 28, 2466 (1985).

[16] A. Brizard, Phys. Fluids B 4, 1213 (1992).

[17] G. W. Hammett, W. Dorland, and F. Perkins, Phys. Fluids B 4, 2052 (1992).

[18] W. Dorland and G. W. Hammett, Phys. Fluids B 5, 812 (1993).

[19] A. Zeiler, J. F. Drake, and B. Rogers, Phys. Plasmas 4, 2134 (1997).

[20] X. Xu, R. H. Cohen, T. D. Rognlien, and J. R. Myra, Phys. Plasmas 7, 1951 (2000).

[21] P. B. Snyder and G. W. Hammett, Phys. Plasmas 8, 3199 (2001).

[22] A. Hasegawa and K. Mima, Phys. Rev. Lett. 39, 205 (1977).

[23] P. J. Morrison, Rev. Mod. Phys. 70, 467 (1998).

[24] R. D. Hazeltine, C. T. Hsu, and P. J. Morrison, Phys. Fluids 30, 3204 (1987).

[25] F. L. Waelbroeck, R. D. Hazeltine, and P. J. Morrison, Physics of Plasmas 16, 032109 (2009).

[26] F. L. Waelbroeck, J. T. M. Antonsen, P. N. Guzdar, and A. B. Hassam, Physics of Fluids B: Plasma Physics 4, 2441 (1992).

[27] N. Winsor, J. L. Johnson, and J. M. Dawson, Physics of Fluids 11, 2448 (1968).

[28] K. Itoh, K. Hallatschek, and S.-I. Itoh, Plasma Physics and Controlled Fusion 47, 451 (2005).

[29] S. Vincena, W. Gekelman, and J. Maggs, Phys. Rev. Lett. 93, 105003 (2004).

[30] C. T. Hsu, R. D. Hazeltine, and P. J. Morrison, Phys. Fluids 29, 1480 (1986).

[31] E. Tassi, P. J. Morrison, F. L. Waelbroeck, and D. Grasso, Plasma Physics and Controlled Fusion 50, 085014 (29pp) (2008).

[32] P. J. Morrison and R. D. Hazeltine, Phys. Fluids 27, 886 (1984).

[33] D. Grasso, F. Califano, F. Pegoraro, and F. Porcelli, Phys. Rev. Lett. 86, 5051 (2001).

[34] J. L. Thiffeault and P. J. Morrison, Physica D 136, 205 (2000). 
[35] O. Izacard, C. Chandre, E. Tassi, and G. Ciraolo, Gyromap for a two-dimensional hamiltonian fluid model derived from Braginskii's closure for magnetized plasmas, submitted to Phys. Plasmas, 2011.

[36] F. L. Waelbroeck, Nuclear Fusion 49, 104025 (15pp) (2009).

[37] F. L. Waelbroeck, F. Militello, R. Fitzpatrick, and W. Horton, Plasma Physics and Controlled Fusion 51, 015015 (22pp) (2009). 